\title{
The role of postoperative chemotherapy in patients who undergoing surgery following chemoradiotherapy of initially unresectable rectal cancer
}

\author{
Michał Jankowski ${ }^{1,2}$, Manuela Las-Jankowska ${ }^{1,3}$, \\ Dariusz Bała ${ }^{1,2}$, Wojciech Zegarski ${ }^{1,2}$
}

Introduction. Preoperative chemoradiotherapy (preCRT) improves local control of rectal cancer and such is particularly merited for treating locoregionally advanced tumors. Nevertheless, the role of postoperative chemotherapy (postCT) in such patients is currently disputed.

Materials. Subjects were 75 patients with unresectable cT3-4 and/or N+ tumors who underwent radical surgery following preCRT between January 2003 and December 2012 at the Oncology Centre in Bydgoszcz. PostCT was subsequently used in 32 (43\%) of these patients.

Results. There were 20 abdominoperineal resections (APR), 50 anterior resections (AR) and 5 Hartmann's procedures (HART) performed in the patient group, where respectively $30 \%, 46 \%$ and $60 \%$, received systemic treatment. Based on postoperative histopathological assessment, disease staging was assigned as follows: stage III for 32 (43\%), patients,stage II for 22 (29\%) and stage I for 15 (20\%). Pathologic complete pathological response (pCR) was seen in 6 cases (8\%). In the postCT+ group, disease stage III was observed in 13 (41\%) patients. A three-year survival was observed in 43 patients; 25 (58\%) and 18 (56\%) of patients respectively undergoing either postCT- or postCT+. A five-year survival was noted in 26 patients; 19 (44\%) and 7 (22\%) in both groups, respectively.

Conclusion. Together with the most recent reports, our study demonstrates that postoperative chemotherapy has no significant effect on the outcomes of oncological treatment in those patients having undergone preoperative chemoradiotherapy for locoregionally advanced rectal cancer.

NOWOTWORY J Oncol 2017; 67, 5: 281-284

Key words: rectal cancer, postoperative chemotherapy, preoperative chemoradiotherapy, unresectable rectal cancer

\section{Introduction}

Rectal cancer (ICD 10: c20) is the most common location of colorectal cancer, of which in 2013, there were 5898 people diagnosed with this condition in Poland [1]. Therapy is determined by the disease stage upon diagnosis and invoIves a number of surgical procedures, as well as radio- and chemotherapies. In locoregionally advanced tumors, the most common treatment involves a surgical resection and radiation therapy combined with systemic treatment. It is therefore crucial that preoperative diagnostics and eligibility for treatment (MRI, CT) be appropriately applied to patient diagnosis and treatment.

\section{Methods \\ "Unresectable rectal cancer"}

This should be diagnosed before surgery, when performing radical resection is fraught with uncertainty due to the risk of positive surgical margins. The tumor is most common-

${ }^{1}$ Chair of Surgical Oncology, Ludwik Rydygier Collegium Medicum in Bydgoszcz, Nicolaus Copernicus University in Toruń, Poland

${ }^{2}$ Department of Surgical Oncology, Oncology Center — Prof. Franciszek Łukaszczyk Memorial Hospital, Bydgoszcz, Poland ${ }^{3}$ Department of Clinical Oncology, Oncology Center — Prof. Franciszek Łukaszczyk Memorial Hospital, Bydgoszcz, Poland 
ly immobile during rectal examination (Tab. I). Pelvic MRI scans are the most efficacious means for diagnosing rectal tumors. An "unresectable rectal cancer" without distant metastases occurs in about $10 \%$ of patients with rectal cancer.

\section{Preoperative treatment}

Perioperative radiotherapy reduces the local recurrence rate in patients with rectal cancer $[2,3]$. When used prior to the main treatment, it also allows the desired outcome to be achieved with a lower toxicity [4]. Reduced tumor size is usually observed several weeks after radiotherapy is completed $[5,6]$. Combining chemo- and radiotherapy increases treatment efficacy [7].

The treatment regimen for the study group consisted of 28 fractions of 1.8-2 Gy per fraction and a 5.4 Gy bolus.

\section{Postoperative chemotherapy}

This has been used since the 1970s, after studies had been published on its efficacy in dealing with colonic cancer patients; being based on fluoropyrimidine [8]. Such therapy showed that the overall survival (OS) and disease-free survival (DFS) rates could be improved by more than 10\% [9].

Rectal cancer patients are qualify for this treatment because the indications are similar to those of colon cancer. The standard duration lasts about 24 weeks, with the treatment being well tolerated, with the most important of the side effects being cardiotoxicity affecting its therapeutic tolerance.

In recent years using postoperative chemotherapy in patients with rectal cancer, particularly following earlier preoperative chemoradiotherapy, is widely considered controversial and is disputed.

\section{Prognosis}

Prognoses for rectal cancer patients, i.e. overall survival, (OS), disease free survival (DFS), depends on how radical resection (R0) is performed with total mesorectal excision (TME) [10]. Locoregionally advanced rectal cancer (cT3-4 and/or $\mathrm{cNO} /+$ ) is associated with higher local recurrence rates following surgical curative treatment during the postoperative period. Preoperative chemoradiotherapy (preCRT) facilitates local control over rectal cancer; increasing the numbers of $\mathrm{R} 0$ resections and decreasing local recurrence rates. This type of treatment is especially justified for locoregionally advanced tumors.

Table I. "Unresectable" rectal cancer

\begin{tabular}{l}
\hline At least one of the following criteria: \\
\hline Immobile upon per rectum examination \\
Adhesion or infiltrating adjacent organs (cT4) \\
Neoplastic infiltration of mesorectal fascia sized $1 \mathrm{~mm}$ or smaller \\
\hline
\end{tabular}

Despite the progress achieved in local and regional treatment, the final outcomes in rectal cancer are still governed by the $\approx 25 \%$ rate of distant metastases following radical resection.

\section{Material}

Subjects were 787 patients with WHO stage I-III rectal tumors that underwent radical surgery between 2003-2012 at the Department of Oncological Surgery of the Nicolaus Copernicus University Medical Centre, Centre of Oncology in Bydgoszcz. Seventy-five patients were preoperatively diagnosed (by computed tomography/magnetic resonance of the pelvis and abdomen) with cT3-4 and/or cNO/+ stage and qualified for preoperative chemoradiotherapy (28 fractions: $25 / 1.8 \mathrm{~Gy}$, and bolus $5.4 \mathrm{~Gy}$, combined with 2 cycles of 5 Fu with leucovorin).

After surgery, postoperative chemotherapy (post-CT+) was administered to 32 patients while 43 did not undergo any postoperative systematic treatment (post-CT-). In the former group, 13 patients did not receive the complete treatment (8 cycles 5Fu/Leu, 40\%). Table II presents the characteristics of these two study groups.

The reasons for abandoning the systemic treatment (post-CT-) are listed in Table III.

\section{Results}

There were no statistically significant differences found when comparing 5-year overall survival (OS) rates between both groups as estimated by the Kaplan-Meier method (Fig. 1). Nor were any such differences observed in either of the 5-year disease-free survival (DFS) rates.

Table II. Patients' characteristics $(n=75$

\begin{tabular}{lccc}
\hline & post-CT+ & post-CT- & Total \\
\hline Above 75 years of age & 4 & 6 & \\
Below 75 years of age & 28 & 37 & \\
AR & & & 50 \\
APR & & & 20 \\
HR & & 5 \\
I/II & 19 & 24 & 33 \\
III & 13 & 19 & 32 \\
\hline
\end{tabular}

Table III. Causes for abandoning adjuvant chemotherapy (post-CT-)

\begin{tabular}{lc}
\hline Cause & ( $\mathrm{n}=43)$ \\
\hline Inadequate qualifications of the oncologist - age & 5 \\
Inadequate qualifications of the oncologist - other & 12 \\
Extension of postoperative period - complications & 6 \\
Extension of postoperative period - other & 4 \\
Other & 4 \\
Unknown & 12 \\
\hline
\end{tabular}


Table IV. Treatment outcomes

\begin{tabular}{lccc}
\hline & post-CT+ & post-CT- & Total \\
\hline $\mathrm{n}$ & 32 & 43 & 75 \\
\multirow{2}{*}{ Local recurrence } & 5 & 6 & 11 \\
& $15.6 \%$ & $13.9 \%$ & $14.7 \%$ \\
\hline
\end{tabular}

The overall $(n=75)$ rate of local recurrences was $14 \%$, without any statistically significant difference between groups (Tab. IV). The local recurrence rate was higher in groups at the lower tumor stages by $4.2 \%$ [11].

\section{Discussion}

\section{The effect of postoperative chemotherapy on overall survival (OS) and disease-free survival (DFS) in patients after preoperative chemoradiotherapy}

Indications for postoperative chemotherapy in patients undergoing radical treatment of rectal cancer are largely based on the outcomes of colonic cancer treatment which show a $24-33 \%$ reduced risk of death as a result of post-CT in patients with regional metastases $(\mathrm{pN}+)$ [12].In their analysis mainly based on studies prior to 2000 and without the use of the TME technique, Petersen et al. reported relative improvements in OS and DFS following post-CR, respectively amounting to $17 \%$ and $25 \%$ [13].

Nevertheless adjuvant chemotherapy in rectal cancer patients after previous preoperative therapy has been the subject of a significant dispute/controversy in recent years.

In their meta-analysis based on 4 studies [14-17] and the data from 1196 patients, Breugom et al. [18] demonstrated that postoperative chemotherapy had no effect on improving overall survival (OS), nor disease-free survival (DFS) or on the rates of all recurrences.

Similar results were published by Bujko et al. in their meta-analysis based on similar study material [19].

Nonewithstanding In addition, Bujko et al. observed a beneficial effect of postoperative chemotherapy on DFS $(2.5 \%$ difference in 5-year DFS rates; HR $=0.92,95 \% \mathrm{Cl}: 0.80-1.04$, $p=0.047)$, with no effect on 5 -year OS rates $(p=0.39)$ in trials conducted on patients randomized after surgical treatment (PROCTOR/SCRIPT; CHRONICLE; QUASAR) [16, 17, 20]. For studies on patients randomized before preoperative radiotherapy (EORTOC, ITALIAN) [14, 15], no effect of postoperative chemotherapy could be observed regarding either DFS or OS rates.

\section{The importance of the distance between the tumor and the anorectal line}

When tumors are located within the upper part of rectum $(10-15 \mathrm{~cm})$, postoperative chemotherapy beneficially affects disease free survival and the incidence of distant metastases [14]. However, one should keep in mind that for tumors located within the upper part of the rectum (more than $10 \mathrm{~cm}$ from the anorectal line), preoperative radiation should be administered only in exceptional cases [21].

An analysis by the Swedish Cancer Registry based on 436 patients aged above 75 years suffering regional disease (WHO stage III), confirms that postoperative chemotherapy is effective only in tumors located more than $10 \mathrm{~cm}$ above the anorectal line (HR 0.54; 95\% Cl 0.3-0.9, p < 0.05) [22]. Such results may be explained by the similarities in anatomical structure of the colon and the upper rectum.

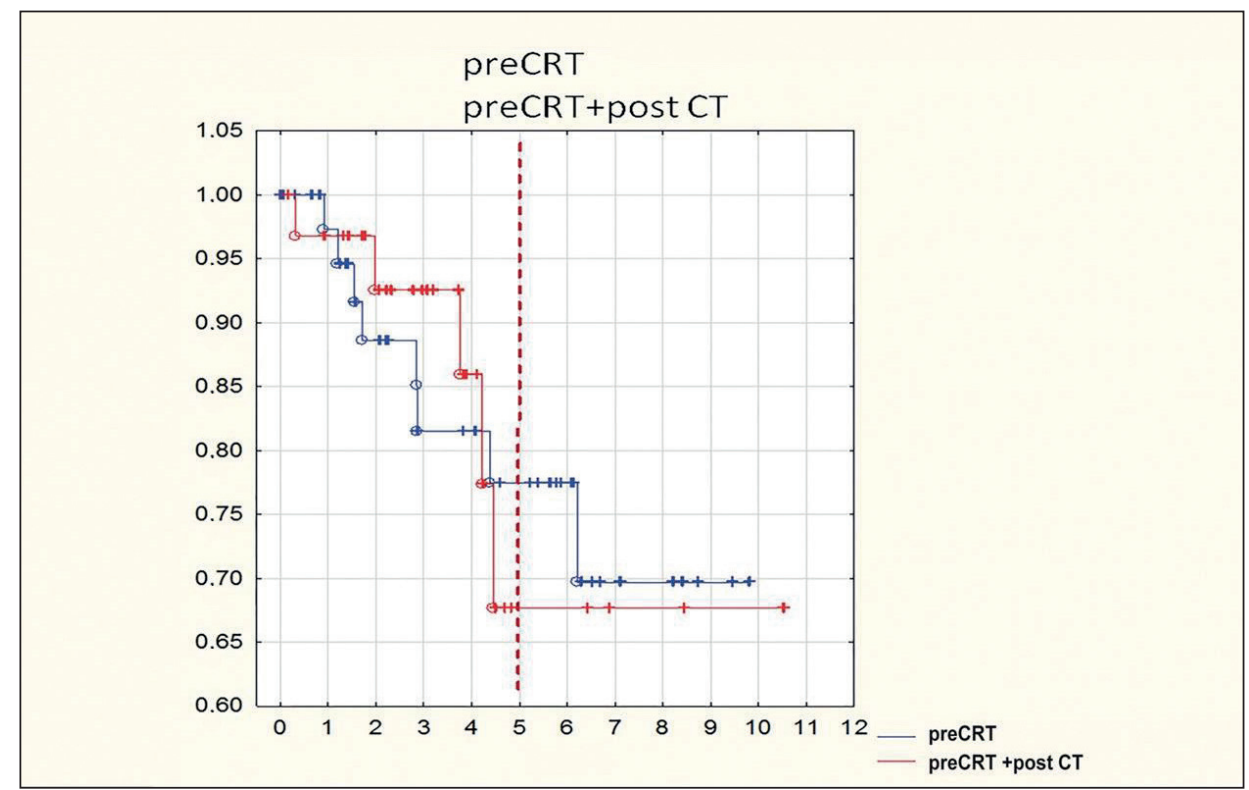

Figure 1. Overall survival (OS) in patients from both groups (with and without postoperative chemotherapy) 


\section{Locoregional advanced}

In locoregionally advanced (cT3-4, mrf+) and/or regionally advanced (cN+), the most effective treatment invoIves preoperative radiotherapy, if possible combined with chemotherapy and a radical (R0) resection procedure by means of the total mesorectal excision (TME) technique [21]. Despite such management, the rates of recurring disease are significantly higher in this group [23]. Correct preoperative diagnostics, preferably by means of pelvic magnetic resonance imaging [24] is thus the crucial element of patient diagnosis that facilitates customization of treatment.

In the retrospectively analyzed group, postoperative chemotherapy was found not to improve oncological outcomes (OS, DFS) despite that the comparison was conducted between apatient group treated according to optimum principles (post- $\mathrm{CT}+$ ) and a heterogeneous patient group in whom systemic treatment was abandoned (post-CT-). A randomized trial is thereby needed to confirm that systemic treatment has no effect overall outcomes.

\section{Conclusions}

Despite abandoning postoperative chemotherapy, OS and DFS rates were not reduced in patients undergoing radical surgical treatment following preoperative chemoradiotherapy of locoregional advanced rectal cancer.

\section{Conflict of interest: none declared}

\section{Michał Jankowski, MD, PhD}

Nicolaus Copernicus University

Collegium Medicum

Oncology Centre — Prof. Franciszek Łukaszczyk Memorial Hospital

Chair of Surgical Oncology

ul. Romanowskiej 2

85-796 Bydgoszcz, Poland

e-mail:michaljankowski@post.pl

Received: 14 Jun 2017

Accepted: 18 Sept 2017

\section{References}

1. Krajowy Rejestr Nowotworów, http://onkologia.org.pl/k/epidemiologia/, 2017.08.30.

2. Local recurrence rate in a randomised multicentre trial of preoperative radiotherapy compared with operation alone in resectable rectal carcinoma. Swedish Rectal Cancer Trial. Eur J Surg 1996; 162 : 397-402.

3. Kapiteijn E, Marijnen CA, Nagtegaal ID et al. Dutch Colorectal Cancer Group. Preoperative radiotherapy combined with total mesorectal excision for resectable rectal cancer. N Engl J Med 2001; 345: 638-646.

4. Glimelius B, Holm T, Blomqvist L. Chemotherapy in addition to preoperative radiotherapy in locally advanced rectal cancer - a systematic overview. Rev Recent Clin Trials 2008; 3: 204-211.

5. Marijnen CA, Nagtegaal ID, Klein Kranenbarg E et al.; Pathology Review Committee and the Cooperative Clinical Investigators. No downstaging after short-term preoperative radiotherapy in rectal cancer patients. J Clin Oncol 2001; 19: 1976-1984.

6. Francois $\mathrm{Y}, \mathrm{Nemoz} \mathrm{CJ}$, Baulieux J et al. Influence of the interval between preoperative radiation therapy and surgery on downstaging and on the rate of sphincter-sparing surgery for rectal cancer: the Lyon R90-01 randomized trial. J Clin Oncol 1999; 17: 2396.

7. Bosset JF, Collette L, Calais G et al. EORTC Radiotherapy Group Trial 22921. Chemotherapy with preoperative radiotherapy in rectal cancer. N Engl J Med 2006; 355: 1114-1123.

8. Moertel CG, Fleming TR, Macdonald JS et al. Levamisole and fluorouraci for adjuvant therapy of resected colon carcinoma. N Engl J Med 1990; 322: 352-358.

9. Gill S, Loprinzi CL, Sargent DJ et al. Pooled analysis of fluorouracil-based adjuvant therapy for stage II and III colon cancer: who benefits and by how much? J Clin Oncol 2004; 22: 1797-1806.

10. Heald RJ, Ryall RD. Recurrence and survival after total mesorectal excision for rectal cancer. Lancet 1986; 1: 1479-1482.

11. Jankowski M, Las-Jankowska $D$, Bala et al. Local recurrence after neoadjuvant radiotherapy resectable locoregional advanced rectal cancer. Eur J Surg Oncol 2016; 42: S150-151. Proceedings of the 36th Congress of the ESSO, 14-16 Sept 2016, Kraków, Poland.

12. Efficacy of adjuvant fluorouracil and folinic acid in colon cancer. International Multicentre Pooled Analysis of Colon Cancer Trials (IMPACT) investigators. Lancet 1995; 345: 939-944.

13. Petersen $\mathrm{SH}$, Harling $\mathrm{H}$, Kirkeby LT et al. Postoperative adjuvant chemotherapy in rectal cancer operated for cure. Cochrane Database Syst Rev 2012: CD004078.

14. Breugom AJ, Swets M, Bosset JF et al. Adjuvant chemotherapy after preoperative (chemo)radiotherapy and surgery for patients with rectal cancer: a systematic review and meta-analysis of individual patient data. Lancet Oncol 2015; 16: 200-207.

15. Bosset JF, Calais G, Mineur L et al.; EORTC Radiation Oncology Group Fluorouracil-based adjuvant chemotherapy after preoperative chemoradiotherapy in rectal cancer: long-term results of the EORTC 22921 randomised study. Lancet Oncol 2014; 15: 184-190.

16. Sainato A, Cernusco LNV, Valentini V et al. No benefit of adjuvant fluorouracil leucovorin chemotherapy after neoadjuvant chemoradiotherapy in locally advanced cancer of the rectum (LARC): Long term results of a randomized trial (I-CNR-RT). Radiother Oncol 2014; 113: 223-229.

17. Glynne-Jones R, Counsell N, Quirke P et al. Chronicle: results of a randomised phase III trial in locally advanced rectal cancer after neoadjuvant chemoradiation randomising postoperative adjuvant capecitabine plus oxaliplatin (XELOX) versus control. Ann Oncol 2014; 25: 1356-1362.

18. Breugom AJ, van Gijn W, Muller EW et al. Adjuvant chemotherapy for rectal cancer patients treated with preoperative (chemo)radiotherapy and total mesorectal excision: a Dutch Colorectal Cancer Group (DCCG) randomised phase III trial. Ann Oncol 2015; 26: 696-701.

19. Bujko K, Glimelius B, Valentini V et al. Postoperative chemotherapy in patients with rectal cancer receiving preoperative radio(chemo)therapy: A meta-analysis of randomized trials comparing surgery \pm a fluoropyrimidine and surgery + a fluoropyrimidine \pm oxaliplatin. Eur J Surg Oncol 2015; 41: 713-723.

20. QUASAR Collaborative Group. Adjuvant chemotherapy versus observation in patients with colorectal cancer: a randomised study. Lancet 2007; 370: 2020-2029.

21. Schmoll HJ, Van Cutsem E, Stein A et al. ESMO Consensus Guidelines for management of patients with colon and rectal cancer. A personalized approach to clinical decision making. Ann Oncol 2012; 23: 2479-2516.

22. Tiselius C, Gunnarsson U, Smedh K et al. Patients with rectal cancer receiving adjuvant chemotherapy have an increased survival: a population-based longitudinal study. Ann Oncol 2013; 24: 160-165.

23. Al-Sukhni E, Milot L, Fruitman M et al. Diagnostic accuracy of MRI for assessment of $T$ category, lymph node metastases, and circumferential resection margin involvement in patients with rectal cancer: a systematic review and meta-analysis. Ann Surg Oncol 2012; 19: 2212-2223.

24. Bujko K, Wyrwicz L, Rutkowski A et al.; Polish Colorectal Study Group. Long-course oxaliplatin-based preoperative chemoradiation versus $5 \times 5$ Gy and consolidation chemotherapy for CT4 or fixed cT3 rectal cancer: results of a randomized phase III study. Ann Oncol 2016; 27: 834-842. 